\section{Modulating protein stability}

Many industrial and research biotechnology applications depend on predictable, controllable expression of exogenous proteins. On page 1298, Stack et al. describe a method that allows regulation of the half-life of proteins of interest. The ubiquitin-proteasome pathway is responsible for the degradation of the great majority of cellular proteins. The authors manipulated this pathway by expressing proteins of interest as fusions with a mutant form of ubiquitin that is recognized as a degradation signal and targets proteins for proteasomal degradation. They show that increasing the number of ubiquitin units leads to a graded decrease in half-life and steady-state levels when fused to reporter proteins as well as cellular proteins, allowing fine-tuning of protein stability. Such fine-tuning could have diverse applications, such as modulating the accumulation of metabolic intermediates by altering key regulatory molecules in metabolic and signaling pathways. They also demonstrated that cells expressing a destabilized $\beta$-lactamase reporter could potentially function as a highthroughput model system for monitoring proteasomal degradation in living cells.

$M S$

\section{A map of the protein world}

In this issue, Schwikowski et al. (see p. 1257) compile 2,709 protein-protein interactions from the yeast community and determine that these interactions form a large network of 2,358 interactions among 1,548 proteins, and several smaller networks. This map provides an intriguing starting point for assigning functions to uncharacterized proteins, by tracing their interacting partners back to proteins with known activities. It also provides a glimpse into how protein interactions can unexpectedly span cellular processes and compartments. The map will take on added dimensions as the functions of now uncharacterized proteins are added, and as the approach is applied to other organisms, allowing cross-referencing of protein interactions across a spectrum of species (see also p. 1242).

This Month in Nature Biotechnology written by Natalie DeWitt, Judy Jamison, Andrew Marshall, and Meeghan Sinclair.

\section{Evolving antibodies in a test tube}

In this issue, Hanes et al. use ribosome display, a technology previously developed for generating diverse protein libraries, to evolve high-affinity antibodies in a cell-free system. Ribosome display involves transcription and translation of libraries in such a way that the transcript, ribosome, and protein product remain physically linked. In this study they applied the technology to evolve and affinity-mature a large synthetic naïve human single-chain antibody ( $\mathrm{scFv}$ ) library. First they translated the scFv library and selected scFvs that bound to insulin. Next the mRNA encoding the bound $\mathrm{scFv}-$ and still associated with it-was released and subjected to affinity maturation, which involved several more rounds of reverse transcription, error-prone PCR, translation, and selection. The procedure yielded scFvs with picomolar affinities in just a few days, and without use of a single living organism (see p. 1287).

$N D$

\section{Gene regulation in flux}

Some applications of metabolic engineering, particularly those in which a high rate of conversion of substrate to product is needed, require control of flux through the central carbon metabolic pathway. This can be problematic, because it requires increased activity of many enzymes in the pathway, and regulation of the pathway is rigid. On page 1283, Ostergaard et al. apply a strategy to uniformly increase the enzyme activities through the yeast $G A L$ pathway by engineering the regulatory networks for gene expression-reducing the levels of negative regulators and increasing levels of positive activators. In this case, by eliminating three known negative regulators of the GAL pathway, Gal6, Gal80, and Mig1, they could increase flux through the galactose utilization pathway by $41 \%$. ND

\section{Ramping up antigen presentation}

To be effective in cancer immunotherapy, tumor antigens must be efficiently processed and presented to $\mathrm{T}$ cells by antigen-presenting cells (APCs), of which dendritic cells are considered to be the most potent. On page 1273 Irvine et al. present a way to efficiently transfect dendritic cells ex vivo using a cationic peptide to condense and mediate cell uptake of a DNA construct encoding the antigen. They go on to show that dendritic cells transfected in this manner effectively stimulate $\mathrm{T}$ cells to develop antigenspecific cytotoxic T-lymphocyte (CTL) activity. Mice injected with the transfected dendritic cells developed antigen-specific immune responses and fended off tumors more robustly than mice injected with dendritic cells pulsed with the antigen peptide.

In a related study, Laus et al. focused on improving uptake of extracellular antigens into APCs for vaccine development. Such soluble antigens are notoriously poor at stimulating CTL responses because they have difficulty gaining entry to APCs that process and present them to the CTLs. Laus et al. have overcome this barrier by adding chargemodifying peptide sequences to the antigen to counteract the repulsive cell surface charge and promote endocytosis of the antigen. They also coupled the antigen to a fusogenic peptide that disrupts the endosomal membrane to release the antigen into the cytosol, making it more accessible to the MHC class I antigen presentation machinery, and boosting the levels of specific CTL immunity in vivo (see p. 1269).

$M S$

On page 1262 Selinger et al. provide the first detailed report of Escherichia coli global gene expression analysis using DNA oligonucleotide microarray chips. The chips used in the study were designed with dense coverage of oligonucleotide probes over the entire genome so that both open reading frames and intergenic regions could be intensively sampled, and so that both strands of the genome are represented. They used the chips to compare gene expression in log versus stationary phase, and the results both agree with the literature and identify novel sets of co-regulated genes. The sub-transcript-level resolution also allows for analysis of operon structure, identification of small RNAs and antisense RNAs, and some aspects of RNA secondary structure (see also p. 1241). 